\title{
Cost-effectiveness of combination LMWHs/UFH versus UFH/LMWHs for the prevention of postsurgical venous thromboembolism at orthopedic department in Clinical Hospital Stip
}

\author{
Biljana Lazarova ${ }^{1}$, Zorica Naumovska², Aleksandra Kapedanovska Nestorovska ${ }^{2}$, \\ Zoran Sterjev², Ljubica Suturkova ${ }^{2}$ \\ ${ }^{1}$ Clinical hospital, Ljuben Ivanov bb, 2000 Stip, N. Macedonia \\ ${ }^{2}$ Faculty of Pharmacy, Ss Cyril and Methodius University, Mother Theresa 47, 1000 Skopje, N. Macedonia
}

\section{Introduction}

Venous thromboembolism (VTE), which includes both deep vein thrombosis (DVT) and pulmonary embolism (PE), is a major burden on the health care system. The risk of VTE is particularly high in patients who undergo major orthopedic surgical interventions, especially interventions for total hip or knee replacement due to perioperative activation of blood coagulation, the effects of surgical trauma of the femoral and iliac vein or embolism due to prolonged bed stay (Imberti et al., 2011). Consequences of VTE and its long-term complications can significantly impair the quality of life in terms of patient health, while the treatment of the condition and recurrent complications become significant costs for the health care provider. Costs are also made during the period of hospitalization immediately after surgical interventions for total hip replacement (THR) and total knee replacement (TKR), as well as in months after discharge from the hospital. Thromboprophylaxis significantly reduces the risk of perioperative VTE. The longer duration of thromboprophylaxis, the lower incidence of VTE. Without anticoagulant prophylaxis, about $50 \%$ of patients with symptomatic proximal DVT or PE have a recurrent thrombosis within three months (Torbicki et al., 2008). The most frequently recommended VTE prophylaxis in the 2004 ACCP consensus guidelines is lowmolecular-weight heparin (LMWH) or unfractionated heparin (UFH).

The updated recommendations in 2008, released by ACCP include fondaparinux alongside LMWH and UFH for the prevention of VTE in certain patient populations (Geerts et al., 2008). There is no scientifically proven evidence of thromboprophylaxis that begins with UFH and continues with LMWHs and vice versa. During the study period in orthopedic ward 40 patients were identified where thromboprophylaxis was started with one drug and then continued with the other. Having that in consideration we evaluated the cost effectiveness of LMWHs/UFH compared to UFH/LMWHs for the prevention of VTE in this particular patients.

\section{Materials and methods}

In cost-effectiveness analysis the costs are reported in MKD values, and health outcomes are

\footnotetext{
*blazarova@yahoo.com
} 
converted into Quality Adjusted Life Years (QALYs), incorporating the measure of quality of life (utility) in health outcomes. Costeffectiveness between LMWHs/UFH vs. UFH/LMWHs used as thromboprophylaxis in patients underwent orthopedic surgery hospitalized at the orthopedic department in Clinical Hospital Stip was revised. The decision for thromboprophylaxis regime was made by the surgeon's, dependent on the availability of anticoagulants at the hospital pharmacy.

\section{Decision tree model}

A decision tree model was developed (Tree Age Pro 2013 software, Williamstown, MA) in order to evaluate the cost-effectiveness and results of prophylaxis of DVT with LMWHs/UFH v.s UFH/LMWHs in high-risk patients who underwent orthopedic surgery.

\section{Results and discussion}

In the decision tree model analysis, the application of thromboprophylaxis with UFH/LMWHs or LMWHs/UFH combinations were evaluated. The first branching in decision tree has two branches: thromboprophylaxis with LMWHs/UFH, costing 60854.88 MKD and 9.84 QALY per patient, whereas thromboprophylaxis with UFH/LMWHs cost 85605.99 MKD and 70 QALY per patient during hospitalization. Each of the branches was further divided into 4 new branches showing the probability of occurrence or non-occurrence of VTE (DVT and PE). In patients undergoing thromboprophylaxis with the combination LMWHs/UFH the probability of stable condition in patients hospitalized for up to 11 days was 0.991 . The probability of occurrence of DVT is 0.00 and the incidence of PE was 0.009 .

In patients undergoing thromboprophylaxis with a combination of UFH/LMWHs, the probability of stable condition for up to 11 days of hospitalization was 0.915 , and the probability of developing DVT was 0.085 , for PE the probability was 0.00 . Decision tree analysis of combined thromboprophylaxis showed higher cost-effectiveness of LMWHs/UFHs with lower cost, greater efficacy and likelihood of a stable condition being greater than UFH/LMWHs.
The results for combined thromboprophylaxis over a 6-month period showed greater dominance of the LMWHs/UFH combination with an effectiveness of 5.01 QALYs and cost of 30884.76 MKD compared to a combination of UFH/LMWHs where a cost effectiveness of 4.70 QALY is less than 42707Y. ICER is -137204.69 MKD/QALYs. Combined thromboprophylaxis results evaluated in period over a 12-month presented even greater dominance of the LMWHs/UFH combination with an effectiveness of 9.84 QALYs at a cost of 60854.88 MKD compared to a combination of UFH/LMWHs where a greater cost of 85605 of 9.70 QALYs. ICER is -174939.72 MKD/QALYs. The increased cost is 24751.11 MKD and the increased effectiveness is -0.14 QALYs.

\section{Conclusion}

Cost-effectiveness analysis confirmed that LMWHs dominated vs. UFH in major orthopedic surgery, providing greater effectiveness at lower costs. LMWHs also lead to less symptomatic VTE events providing increased QALYs comparing to UFH. The use of LMWHs in this prophylactic indication contributes to the effective use of limited resources, as it is associated with better clinical results at a lower cost.

\section{References}

Geerts, W.H., Bergqvist, D., Pineo, G.F., Heit, J.A., Samama, C.M., Lassen, M.R., Colwell, C.W., 2008. Prevention of venous thromboembolism: American College of Chest Physicians evidence-based clinical practice guidelines (8th edition). Chest 133(Suppl 6), 381S-453S.

Imberti, D., Bianchi, C., Zambon, A., Parodi, A., Merlino, L., Gallerani, M., Corrao, G., 2012. Venous thromboembolism after major orthopaedic surgery: a population-based cohort study. Intern. Emerg. Med. 7(3), 243-249.

Torbicki, A., Perrier, A., Konstantinides, S., Agnelli, G., Galiè, N., Pruszczyk, P., Bengel, F., Brady, A.J., Ferreira, D., Janssens, U., Klepetko, W., Mayer, E., Remy-Jardin, M., Bassand, J.P., ESC Committee for Practice Guidelines (CPG) 2008. Guidelines on the diagnosis and management of acute pulmonary embolism: the task force for the diagnosis and management of acute pulmonary embolism of the

European Society of Cardiology (ESC). Eur. Heart J. 29(18), 2276-2315.

Maced. Pharm. Bull. 66 (Suppl 1) 75 - 76 (2020) 